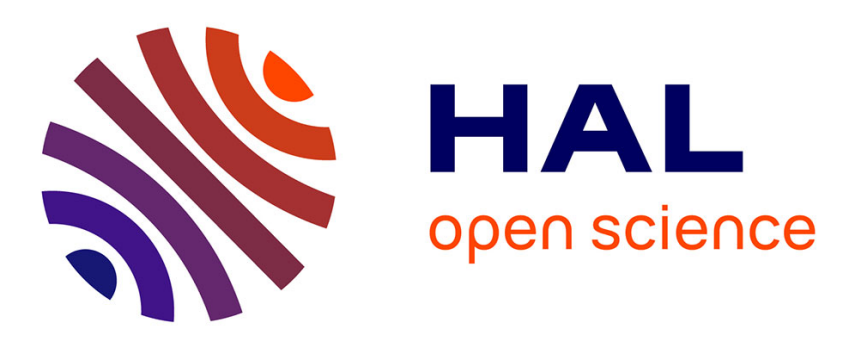

\title{
The effect of Time Scales in Photosynthesis on microalgae Productivity
}

Philipp Hartmann, Quentin Béchet, Olivier Bernard

\section{To cite this version:}

Philipp Hartmann, Quentin Béchet, Olivier Bernard. The effect of Time Scales in Photosynthesis on microalgae Productivity. Bioprocess and Biosystems Engineering, 2014, 37 (1), pp.17-25. 10.1007/s00449-013-1031-2 . hal-00852255

\section{HAL Id: hal-00852255 \\ https://hal.inria.fr/hal-00852255}

Submitted on 20 Aug 2013

HAL is a multi-disciplinary open access archive for the deposit and dissemination of scientific research documents, whether they are published or not. The documents may come from teaching and research institutions in France or abroad, or from public or private research centers.
L'archive ouverte pluridisciplinaire HAL, est destinée au dépôt et à la diffusion de documents scientifiques de niveau recherche, publiés ou non, émanant des établissements d'enseignement et de recherche français ou étrangers, des laboratoires publics ou privés. 


\title{
The effect of Time Scales in Photosynthesis on microalgae Productivity
}

\author{
Philipp Hartmann • Quentin Béchet · \\ Olivier Bernard
}

Received: date / Accepted: date

\begin{abstract}
Microalgae are often seen as a potential biofuel producer. In order to predict achievable productivities in the so called raceway culturing system, the dynamics of photosynthesis has to be taken into account. In particular, the dynamical effect of inhibition by an excess of light (photoinhibition) must be represented. We propose a model considering both photosynthesis and growth dynamics. This model involves three different time scales. We study the response of this model to fluctuating light with different frequencies by slow/fast approximations. Therefore, we identify three different regimes for which a simplified expression for the model can be derived. These expressions give a hint on productivity improvement which can be expected by stimulating photosynthesis with a faster hydrodynamics.
\end{abstract}

\section{Introduction}

Microalgae have received a specific attention in the framework of biodiesel production and renewable energy generation since a decade. Their high actual photosynthetic yield compared to terrestrial plants (which growth is limited by $\mathrm{CO}_{2}$ availability and access to nutrients) could lead to large potential algal biomass productions which is orders of magnitude higher than biofuel from field crops [30].

After a nitrogen limitation, this biomass can reach a very high lipid content (up to $60 \%$ of dry weight [18]). These possibilities have led some authors to consider that microalgae could be one of the main biofuel producers in the future $[14,7]$.

Philipp Hartmann

BIOCORE-INRIA, BP93, 06902 Sophia-Antipolis Cedex, France and UPMC-CNRS LOV, BP28, 06234 Villefranche sur mer, France

E-mail: philipp.hartmann@inria.fr

Quentin Béchet

Massey University, Private Bag 11 222, Palmerston North 4442, New Zealand, E-mail: q.bechet@massey.ac.nz

Olivier Bernard

BIOCORE-INRIA, BP93, 06902 Sophia-Antipolis Cedex, France and UPMC-CNRS LOV, BP28, 06234 Villefranche sur mer, France

E-mail: olivier.bernard@inria.fr 
Moreover, the ability of microalgae to fix $\mathrm{CO}_{2}$ in a controlled way opens up applications in mitigation systems $[1,20]$. Microalgal biofuel production systems could therefore be associated with industrial powerplants with a high $\mathrm{CO}_{2}$ production. In the same spirit, microalgae could be used to consume inorganic nitrogen and phosphorus, and improve wastewater treatment technology [26].

These advantages put microalgae in a good position for renewable energy production at large scale [7]. In the coming years there might be large scale industrial plants to produce microalgae. However, the culture of algae is not straightforward and suffers from many limitations $[22,5]$. As a matter of fact, growth rates in mass cultures are often reduced due to an excess of light which is inhibiting the photosynthesis process. Consequently, productivity is often under its optimal value. Better understanding photoinhibition and therefore improving growth efficiency of the algae is a key issue. The dynamics of photoinhibition has been described by models $[10,11,13]$, but its effect on cell cultures is challenging to predict since photoinhibition is a dynamic mechanism: The level of photoinhibition experienced by a cell does not depend only on the level of light intensity that the cell is exposed to, but as well on the length of exposure. The damaging effect of photoinhibition is counterbalanced by a recovery process that also needs to be considered. In most cultivation systems, the light intensity that cells are exposed to constantly varies as cells move within the culture due to mixing. It should therefore be possible to optimize the hydrodynamics regime in order to minimize the effect of photoinhibition. Another difficulty is that the level of photoinhibition has an impact on the rate of nitrogen uptake, even under nitrogen-limiting conditions. In order to account for this interaction between photoinhibition and nitrogen absorption, this study aims at analyzing the Han photoinhibition dynamics model coupled with the Droop model for nitrogen consumption.

The Droop model $[8,9]$ has been widely studied and proved to accurately reproduce situations of nitrogen limitation [9,25,4]. The Droop model has been validated for the prediction of microalgal growth in the case of nitrogen limitation for biodiesel production [17].

This paper is organized as follows. In a first part, we recall the Droop model. Secondly, we introduce the light influence in this model. In a third part we propose analytic approximations of the model for the whole frequency range. In the last part, the applicability of the model and the approximations are discussed.

\section{The Droop model}

The Droop model has been proven to appropriately represent the effect of macronutrients, such as nitrogen on the growth rate of microalgae [9]. It is known to predict a unique non trivial equilibrium if the culturing conditions (i.e. influent concentration of nitrogen $s_{i n}$ and dilution rate $D$ ) are kept constant $[15,3]$. In contrast to the simpler Monod Model [19], the Droop Model considers a dependence of growth on the intracellular nitrogen concentration or quota $q$. This defines nutrient uptake and growth as uncoupled processes. In the following, the differential equations present a modified version of the Droop Model (as in [2]) representing 
the evolution of the biomass $(x)$ with an inorganic nitrogen substrate $s$.

$$
\text { (D) }\left\{\begin{array}{l}
\dot{s}=D s_{i n}-\rho(s, q) x-D s \\
\dot{q}=\rho(s, q)-\mu(q) q \\
\dot{x}=\mu(q) x-D x
\end{array}\right.
$$

In this model the absorption rate $\rho(s, q)$ and growth rate $\mu(q)$ are generally defined as Michaelis-Menten and Droop functions:

$$
\begin{aligned}
\rho(s, q) & =\rho_{0} \frac{s}{s+K_{s}} \cdot\left(1-q / Q_{l}\right) \\
\mu(q) & =\mu_{0}\left(1-\frac{Q_{0}}{q}\right)
\end{aligned}
$$

where $K_{s}$ is the half saturation constant for substrate uptake and $Q_{0}$ the minimal cell quota. At the quota $Q_{0}$, the growth rate equals 0 . In Eq. (1), a factor $\left(1-q / Q_{l}\right)$ is introduced to account for the down regulation of the uptake when the quota becomes high. It thus limits the internal quota to the following interval [2]:

$$
Q_{0} \leq q \leq Q_{m} \leq Q_{l}
$$

$Q_{m}$ is the maximum cell quota which is obtained in conditions where nutrients are not limiting. As a direct consequence, the growth rate is also bound:

$$
0 \leq \mu(q)<\mu_{\max }=\mu_{0}\left(1-Q_{0} / Q_{m}\right)
$$

where $\mu_{m}$ is the maximum growth rate reached in non limiting conditions. The parameters of the Droop model can be estimated well with growth experiments under nutrient limiting conditions [2]. The Droop model has been widely studied $[15,3,28]$ and validated $[9,25,4,28]$. However, it cannot directly be used for the description of photobioreactors or raceways since it does not account for light.

\section{Integration of the fast photosynthetic unit time scale}

In a microalgae culturing device, the light perception of a cell can vary at a time scale faster than the inherent uptake and growth time scales of the Droop model $[16,21]$. The fast dynamics of the photosynthetic processes within the chloroplasts have thus to be taken into account. On a molecular scale two photosystems types (denoted PSI and PSII) are involved in the photon harvesting process [27]. The photosynthetic production is triggered by the simultaneous excitation of both photocenters. In the Han model [12] it is assumed that the activation of the second photosystem (PSII) is the limiting factor in photosynthetic productivity. These photosystems can be damaged by an excess of energy due to an excess of absorbed photons. The key proteins within the photosynthetic units (PSUs) can be however repaired at a slow rate. Photosystem damage and recovery are the two main processes which drive the dynamics of photoinhibition.

The PSII is assumed to be in one of the three states: open (A), closed (B), or inhibited (C). Open PSUs may be excited by photons, trigger the photosynthetic process, and turn to the closed state. The turnover rate for this process is the 
product of the PSII's cross section $\sigma$ and the light intensity $I$. A closed PSU may return to an open state at the rate $\frac{1}{\tau}$, whereas $\tau$ represents the turnover time of the electron transfer chain. If facing excessive radiation, a closed PSU can also be destroyed by incident light. The rate for photoinhibition processes is defined by the product $k_{d} \sigma I, k_{d}$ being the PSII's damage parameter. Inhibited PSUs can be repaired by chemical processes in the cell at a constant rate $k_{r}$. The equations for the three states (according to [12]) consequently are:

$$
\begin{aligned}
& \frac{d A}{d t}=-I \sigma A+\frac{B}{\tau} \\
& \frac{d B}{d t}=I \sigma A-\frac{B}{\tau}+k_{r} C-k_{d} \sigma I B \\
& \frac{d C}{d t}=-k_{r} C+k_{d} \sigma I B
\end{aligned}
$$

$A, B$ and $C$ are the relative frequencies of the respective states, the sum of $A, B$, and $C$ is therefore 1 and the system can be completely described by two equations only.

To couple this model with the Droop model, we consider that growth rate results from the product of the total cross section of the cell PSUs in open state (resulting thus from their number and size) and light. In line with the Droop model, we assume that the PSUs number and size are related to the internal nitrogen quota with a Droop relationship. In that spirit, the Droop relationship can be seen as a factor describing the total cross section of all PSUs. As a consequence, the total cross section of productive PSUs is assumed to be proportional to $\sigma\left(1-\frac{Q_{0}}{q}\right) A$. The growth rate in terms of inorganic carbon fixation rate is then proportional to the product of the light intensity $I$ with the total cross section of the active photocenters.

$$
\mu(I, A, q)=\mu_{m}\left(1-\frac{Q_{0}}{q}\right) \cdot \sigma I A
$$

where $\mu_{m}$ is a parameter such that $\mu_{m} \sigma I_{0} A=\mu_{0}$ for a constant light $I_{0}$, and the associated fraction of open states $A$.

In the following considerations, only $2 T$-periodic light is studied. Assuming state periodicity, we can compute:

$$
\int_{2 T} \dot{A} d t=-\int_{2 T} I \sigma A d t+\int_{2 T} \frac{B}{\tau} d t=0
$$

Since $A$ is periodic, we have then:

$$
\overline{I A} \sigma=\bar{B} / \tau
$$

where $\overline{I A}$ and $\bar{B}$ denominate the mean values over one period. Following this equation, $\bar{B}$ can be used as an indicator for the photosynthetic productivity; by definition it is bound in the interval $[0,1]$.

$$
\bar{B}=\overline{I A} \sigma \tau
$$

In the following section, we will compute $\bar{B}$ as a function of the light intensity and frequency. Depending on the frequency domains, two approximations $\left(\bar{B}_{\text {slow }}\right.$ and $\left.\bar{B}_{\text {fast }}\right)$ will be given. 


\begin{tabular}{|c|c|c|}
\hline Parameter & Value & Unit \\
\hline$k_{r}$ & $4.8 \cdot 10^{-4}$ & $s^{-1}$ \\
$k_{d}$ & $2.99 \cdot 10^{-4}$ & $\cdot$ \\
$1 / \tau$ & 0.1460 & $s^{-1}$ \\
$\sigma$ & 0.0019 & $\mathrm{~m}^{2} /(\mu \mathrm{mol})$ \\
$I_{0}$ & 2000 & $\mu \mathrm{mol} \mathrm{s} \mathrm{s}^{-1} \mathrm{~m}^{-2}$ \\
\hline
\end{tabular}

Table 1 Model parameters according to [31]

\section{Model analysis}

\subsection{Motivation}

In this section, the mathematical behaviour of the Droop-Han model under periodic forcing of light is investigated. It is worth noting that the time scales associated with the kinetics of light response and nitrogen consumption differ by several orders of magnitude. Indeed, the excitation/relaxation and photodamage/recovery processes have time scales lower than the hour, while the nitrogen uptake has a time scale of hours to days [2]. As a result, by using quasi steady state approximations [24], the analysis of the Droop-Han model will be performed in two steps. First, the light-response will be discussed, and then the effect of nitrogen consumption on this light response will be investigated.

The light response of the model implies two additional time scales of very different magnitude. It can thus be identified as a slow/fast system which can be approximated using singular perturbation theory [24].

\subsection{Light Response}

We rewrite the Han model by using $B=1-A-C$, it is a system with slow/fast time scales:

$$
\begin{aligned}
& \frac{d A}{d t}=-(\sigma I+1 / \tau) A+(1-C) / \tau \\
& \frac{d C}{d t}=k_{d}\left(-\left(k_{r} / k_{d}+\sigma I\right) C+\sigma I(1-A)\right)
\end{aligned}
$$

which can be written in the classical form of a slow/fast system:

$$
\begin{aligned}
\dot{\mathbf{x}} & =\epsilon \cdot(M \mathbf{x}+\mathbf{b}) \\
\epsilon & =\left(\begin{array}{cc}
1 & 0 \\
0 & k_{d}
\end{array}\right) ; M=\left(\begin{array}{cc}
-(\sigma I+1 / \tau) & -1 / \tau \\
-\sigma I & -\left(k_{r} / k_{d}+\sigma I\right)
\end{array}\right) ; \mathbf{b}=\left(\begin{array}{c}
1 / \tau \\
\sigma I
\end{array}\right)
\end{aligned}
$$

Using an exemplary parameter set as shown in Table 1 , the absolute values of the entries of $M$ and $b$ are in the range 0.1 to 6 whereas $k_{d}$ is on the order of $10^{-4}$. This shows that the system of equations $(13-14)$ has a slow/fast dynamics with a time scale ratio of $k_{d}$. The dynamics of $C$ is more than a factor 1000 slower than the dynamics of $A$. Although the considered parameters depend on the species, this key time scale property remains qualitatively true for any species due to the nature of the involved physiological processes. 


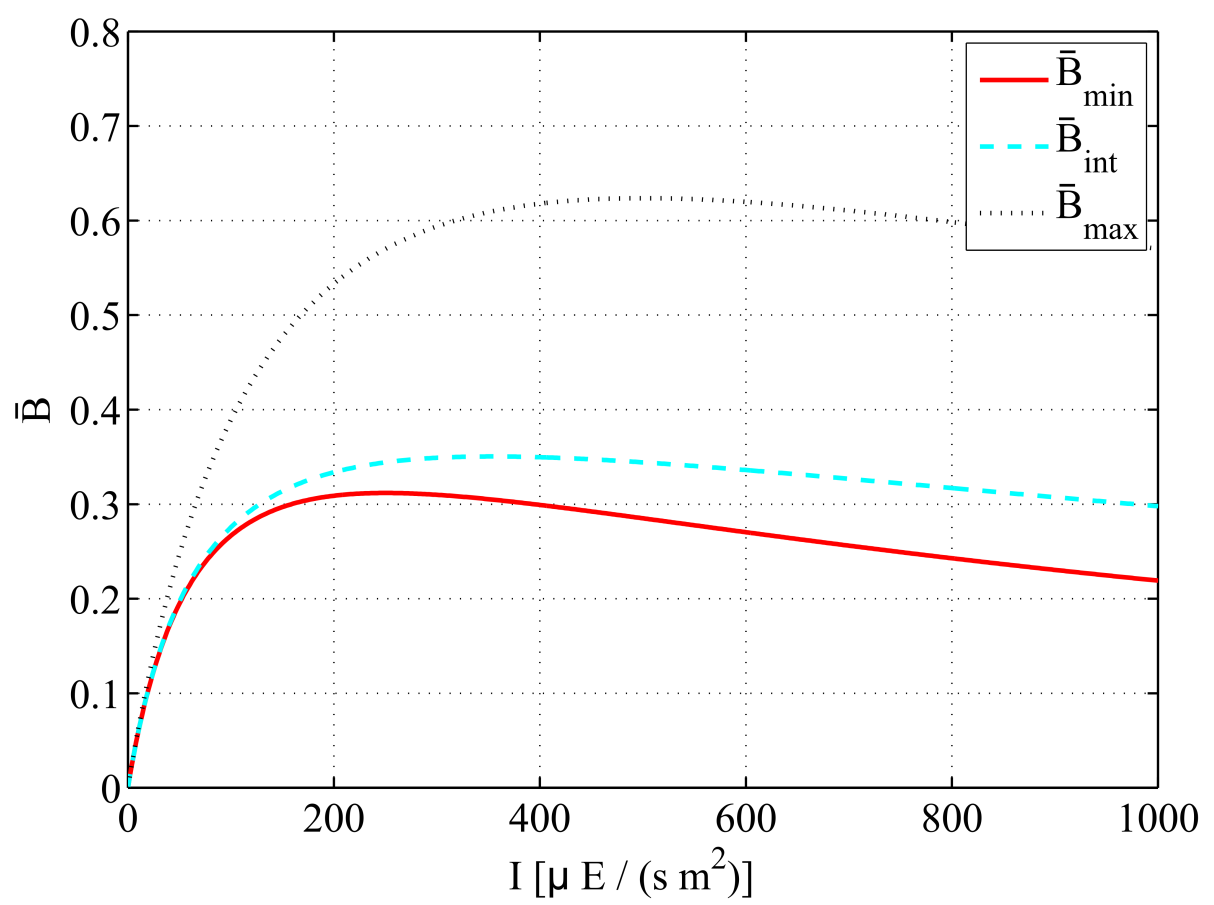

Fig. 1 Direct effect of light on the growth rate: variation of the mean value of $B$ over a period with respect to light intensity. The plot results from the approximations for slow frequency ( $\bar{B}_{\min }$, red), intermediate frequency $\left(\bar{B}_{\text {int }}\right.$, dashed cyan) and fast frequency $\left(\bar{B}_{\max }\right.$, dotted black)

In order to study analytically the model response to fluctuating light, we consider a caricatured light pattern as follows:

$$
I(t)=\left\{\begin{array}{c}
I_{0}, \text { for } 0<\mathrm{t}<\mathrm{T} \\
0, \text { for } \mathrm{T}<\mathrm{t}<2 \mathrm{~T}
\end{array}\right.
$$

Note that the solution of the Han model (14), at steady state, for a continuous light $I_{0}$, can be straightforwardly computed as follows:

$$
\bar{B}_{S S}\left(I_{0}\right)=\frac{\sigma I_{0} \tau}{1+\tau \sigma I_{0}+k_{d} / k_{r} \tau\left(\sigma I_{0}\right)^{2}}
$$

In order to analytically study the model response to various light frequencies, two domains of the light variation frequency have to be distinguished: slow frequencies with $T>10 \cdot \tau \approx 100 \mathrm{~s}$, and fast frequencies with $T \leq 100 \mathrm{~s}$.

In the following paragraphs, the approximations for the low and the high frequency domains are given, as shown in Figures 1 and 2. All approximations consider a forced periodic state of the system. 


\subsubsection{Low frequencies}

For large values of $T$, the fast dynamics of $A$ (Eq. (13)) will reach its slow manifold, given by $(\sigma I+1 / \tau) A=(1-C) / \tau$. The slow dynamics can therefore be represented by Eq. (14), where $A$ is replaced by its expression with respect to $C$. An analytical expression can be derived for $C$ (calculation not shown). The average value along the period $2 T$ can then be determined (see Appendix A1), leading to the following expression for $\bar{B}$ :

$$
\bar{B}_{\text {slow }}=\left(1+\frac{\delta\left(I_{0}\right)}{k_{r}} \frac{1}{T} \frac{(1-\gamma(0))\left(1-\gamma\left(I_{0}\right)\right)}{1-\gamma(0) \gamma\left(I_{0}\right)}\right) \cdot \frac{\bar{B}_{S S}\left(I_{0}\right)}{2}
$$

with

$$
\begin{aligned}
\delta(I) & =\frac{k_{d} \tau \frac{(\sigma I)^{2}}{1+\tau \sigma I}}{k_{r}+\frac{(\sigma I)^{2}}{1+\tau \sigma I}} \\
\gamma(I) & =e^{-\left(k_{r}+k_{d} \frac{(\sigma I)^{2}}{1+\tau \sigma I}\right) T}
\end{aligned}
$$

\subsubsection{High frequencies}

For small $T$, the dynamics of $C$ in Eq. (14) is so slow that $C$ stays approximately constant over one period. As a consequence, Eq. (14) leads thus to a value of $C$ as a function of the mean value of $I$ and $I A$ on one period. This value can then be used in Eq. (13) and defines the dynamics of $B$, as shown in Appendix A2.

$$
\begin{aligned}
\bar{B}_{\text {fast }} & =\frac{\sigma I_{0}}{2} \frac{\eta-\theta+\tau \eta \theta}{\eta^{2}+\frac{k_{d}\left(\sigma I_{0}\right)^{2}}{2 k_{r}}(\eta-\theta)} \\
\eta & =\left(\sigma I_{0}+1 / \tau\right) \\
\theta & =\frac{1}{T} \frac{(1-\alpha(0))\left(1-\alpha\left(I_{0}\right)\right)}{1-\alpha\left(I_{0}\right) \alpha(0)} \\
\alpha(I): & =e^{-(1 / \tau+\sigma I) T}
\end{aligned}
$$

4.3 Monotony of the productivity with respect to light period

Property: The approximated growth rate response composed by $\bar{B}_{\text {fast }}, \bar{B}_{\text {slow }}$ reproduces a continuous and decreasing function of $T$ from $B_{s s}\left(I_{0} / 2\right)$ down to $B_{s s}\left(I_{0}\right) / 2$. The results obtained through this approximation are represented on Fig. 2.

Proof: First, let us show that the successive approximations continuously join up together. Using the rule of de l'Hôpital, the limits of $\bar{B}_{\text {slow }}$ can be estimated for $T \rightarrow 0$ and $T \rightarrow \infty$ :

$$
\begin{aligned}
\lim _{T \rightarrow 0} \bar{B}_{\text {slow }} & =\bar{B}_{\text {int }}=\frac{\sigma I_{0} \tau}{\left(2+2 \tau \sigma I_{0}+k_{d} / k_{r} \tau\left(\sigma I_{0}\right)^{2}\right)} \\
\lim _{T \rightarrow \infty} \bar{B}_{\text {slow }} & =\bar{B}_{\text {min }}=B_{S S}\left(I_{0}\right) / 2
\end{aligned}
$$


The limit for long periodicities is the steady state solution, i.e. 0 during night period and $\bar{B}_{S S}$ during light. Finally, the average response is $\bar{B}_{\min }=B_{S S} / 2$. Considering $\bar{B}_{\text {fast }}$, the same type of calculation leads to:

$$
\begin{aligned}
& \lim _{T \rightarrow 0} \bar{B}_{\text {fast }}=\bar{B}_{\max }=\frac{\tau \sigma \frac{I_{0}}{2}}{\left(1+\tau \sigma \frac{I_{0}}{2}+k_{d} / k_{r} \tau\left(\sigma \frac{I_{0}}{2}\right)^{2}\right)} \\
& \lim _{T \rightarrow \infty} \bar{B}_{\text {fast }}=\bar{B}_{\text {int }}
\end{aligned}
$$

The limit of $\bar{B}_{\text {fast }}$ for low frequencies coincides with the limit of $\bar{B}_{\text {slow }}$ for high frequencies. The limit of $\bar{B}_{\text {fast }}$ for high frequencies is the steady state solution for continuous illumination with the mean intensity $\bar{I}=I_{0} / 2$. As shown in Appendix A3, both approximations $\bar{B}_{\text {fast }}$ and $\bar{B}_{\text {slow }}$ are decreasing functions with respect to $T$. The monotonic behaviour of the functions in repect to $T$ is presented in Fig. 2 .

\subsection{Dynamics of the Droop Variables}

In most cultivation systems, algae are cultivated under nutrient-saturating conditions in order to maximize the productivity. Consequently, we assume $s>>K_{s}$, and the nitrogen absorption rate, as given by Eq. (2) is only a function of $q$. The dynamics of $q$ in system (1) does only depend on $q$, and this stable linear equation can be solved at steady state. The intracellular concentration of nitrogen can be determined, by calculating the equilibrium value of $\mathrm{q}\left(Q_{e q}\right)$ :

$$
Q_{e q}=\frac{\rho_{0}+\mu_{m} \bar{B}(I, T) Q_{0}}{\frac{\rho_{0}}{Q_{l}}+\mu_{m} \bar{B}(I, T)}
$$

where $\bar{B}(I, T)$ is the mean photosynthetic productivity and can stand for any of the presented approximate values. The growth rate can then be computed as follows:

$$
\mu=\mu_{m}\left(1-\frac{Q_{0}}{Q_{e q}(I, T)}\right) \bar{B}(I, T) / \tau=\mu_{H}(I, T)\left(1-\frac{Q_{0}}{Q_{e q}(I, T)}\right)
$$

Figure 3 shows the resulting growth rate $\mu$ as defined in equation (31), the theoretical photosynthetic productivity neglecting any nitrogen limitation $\mu_{H}$ and the equilibrium value for the intracellular nitrogen $Q_{e q}$ as defined in Eq. 30. The function $\bar{B}(I, T)$ was assumed to be equal to $B_{\text {slow }}(I, T)$. As presented, high light conditions lead to a lowered $Q$ and therefore a slight reduction of the growth rate, compared to the photosynthetic efficiency only considered with the Han model. Furthermore, the influence of the steady state value $Q_{e q}$ turns to a constant factor for intermediate and high productivities. Therefore, in the case of non limiting nitrogen, it can be disregarded. 


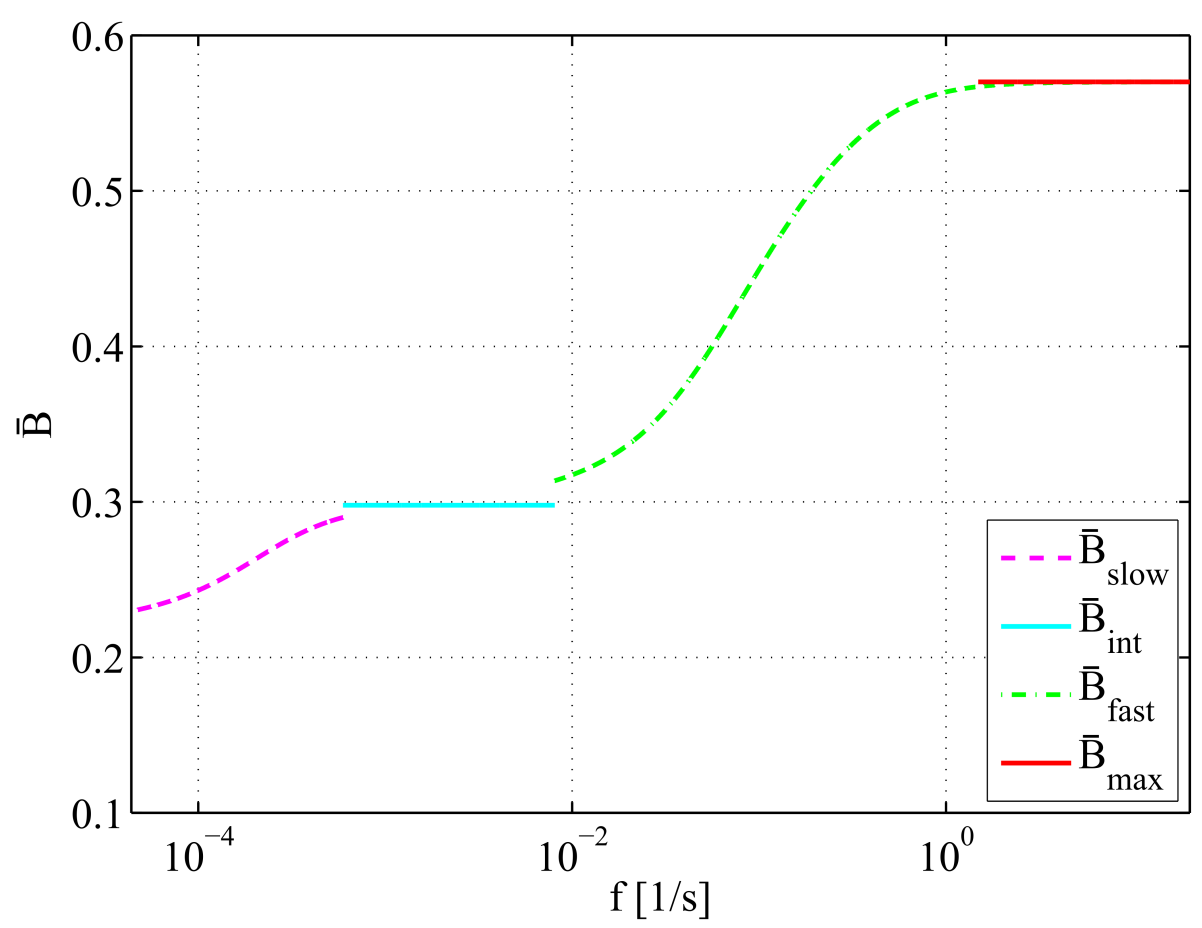

Fig. 2 Approximations of the growth rate over different domains of light frequency $\left(\bar{B}_{\text {slow }}\right.$ : purple dash-line, $\bar{B}_{\text {int }}$ : cyan line, $\bar{B}_{\text {fast }}$ : green dash-line, $\bar{B}_{\text {max }}$ : red-line) against LD-frequency $f=1 / T$ on a logarithmic scale

\subsection{Parameter identification}

From measurements of substrate, biomass and intracellular nitrogen under different conditions, the key parameters of Droop's model can be identified [2]. As it has been discussed, the limited nitrogen quota fluctuation due to light (when nitrogen is not limiting) hardly influences growth rate.

Assuming that the growth rate response for continuous illumination $\bar{B}_{S S}(I)$ (Haldane curve) is available, two parameters can be determined. The parameters $g_{1}$ and $g_{2}$ can be estimated by a least square fit of the function $\bar{B}_{S S}(I)$, as it is defined in eq. (18), normalized by the value of its maximum:

$$
\bar{B}_{S S}=\frac{\sigma I_{0} \tau}{1+g_{1} I_{0}+g_{2} I_{0}^{2}}
$$

with

$$
\begin{aligned}
& g_{1}=\tau \sigma \\
& g_{2}=k_{d} / k_{r} \tau \sigma^{2}
\end{aligned}
$$




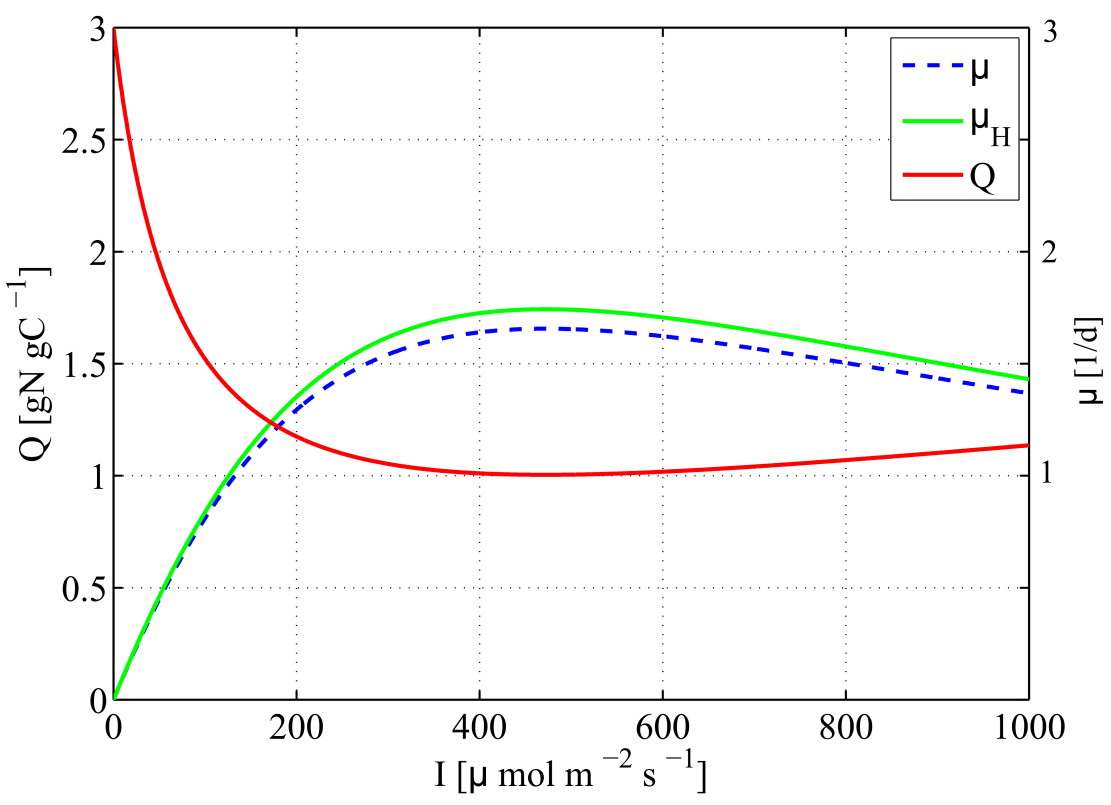

Fig. 3 Intracellular nitrogen ratio at equilibrium $\left(Q_{e q}\right.$, red) in respect to irradiance at steady state for the slow frequency approximation $\bar{B}_{\min }$ (Eq. (30)). The growth rate $\mu$ given by the Droop-Han model (blue dash-line) is compared to the growth rate $\mu_{H}$ computed without the Droop term (green line) (cf. Eq. (31)).

In order to calculate the independent values of $\tau$ and $\sigma$, the following quantity can be calculated for different values of $I_{0}$ :

$$
F_{1}\left(I_{0}\right)=I_{0} \cdot\left(\frac{1}{\bar{B}_{\min }\left(I_{0}\right)}-\frac{1}{\bar{B}_{\text {int }}\left(I_{0}\right)}\right)=\frac{1.5}{\sigma}+1.5 \tau I_{0}
$$

As $F_{1}$ is a linear function of $I_{0}$, a linear regression enables the determination of the values of $\tau$ and $\sigma$. As a consequence, $\sigma, \tau$ and $\frac{k_{d}}{k_{r}}$ can be determined by measuring $\bar{B}_{\text {min }}$ and $\bar{B}_{\text {int }}$ with respect to $I_{0}$ only. To get the absolute values of $k_{d}$ and $k_{r}$, a possible approach is to use the productivity response $\bar{B}_{\text {slow }}$ as a function of $T$. Since the system has only one remaining degree of freedom, a least-square fitting can provide this value.

With the proposed method, measuring the growth rate response for various irradiances and light-dark (LD) frequencies leads to the identification of all the model parameters.

\subsection{Preliminary validation of the Droop-Han Model}

The methodology presented in the previous section is only valid in the case of a simple square signal with light and dark periods of equal length. Furthermore, it is necessary to use diluted cultures to ensure a homogeneous light distribution 


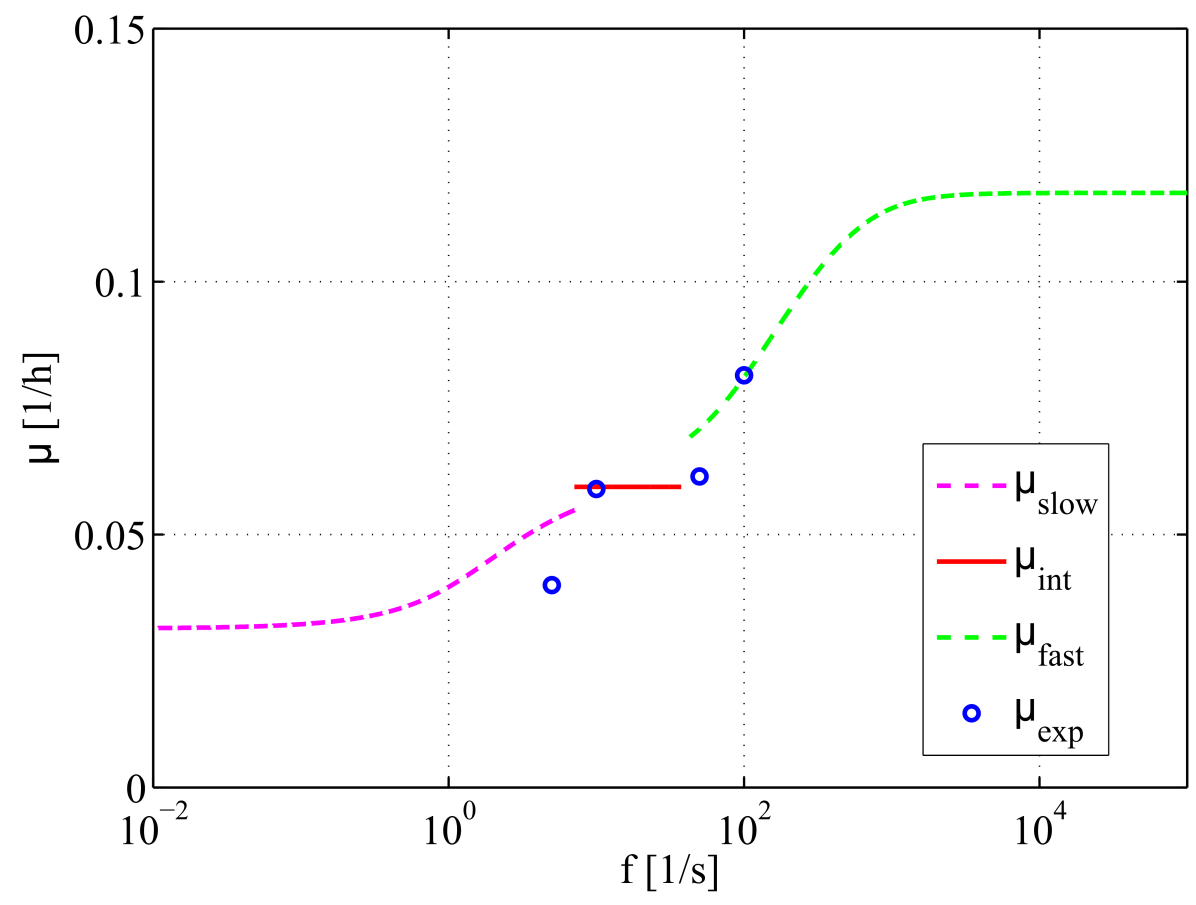

Fig. 4 Growth rate as a function of the frequency of the light signal in the case of a simple square signal (plain lines: theoretical prediction using the Han model; blue circles: experimental data from Vejrazka et al [29]).

within the reactor and avoid an additional flashing effect. In recent experiments, Vejrazka et al. [29] provided data for the growth of Chlamydomonas reinhardtii in LD cycles at a LD ratio of 0.1 . Although, their experimental setup differs from the preconditions in the presented approach, Vejrazka's results are in agreement with the prediction of the Droop-Han model as can be seen in Fig. 4. The "inflexion point" can therefore be identified for this experiment between 10 and $50 \mathrm{~Hz}$. The growth rate for $100 \mathrm{~Hz}$ is equal to the growth rate under continuous light with average light intensity. These results meet very well the predictions of the DroopHan model and its analytical approximations for established periodic regimes. Nevertheless, data covering more variations of $I_{0}$ and $T$ would allow a better model calibration and a more quantitative validation.

\section{Discussion}

With the proposed coupled model, new insights about microalgal response to varying light signals have been obtained. When algae are exposed to periodic flashing light, the Droop-Han model predicts that the growth rate is an increasing function of the light frequency as shown in Fig. 2. It includes plateaus for very low and very high frequencies. A plateau or inflexion point is obtained in a median domain. This specific shape seems to be compatible with the experimental results of Vejrazka et al. [29], supporting then the model validity, as illustrated on Fig. 4. 
The characteristic shape directly illustrates key properties of the Han model: the two gradients in growth rate correspond to the two time scales of the inhibitionrepair process and the activation-relaxation process. For very slow light variations, growth is limited by strong inhibition during the light phase. With increasing frequency, the degree of inhibition turns to a constant equilibrium throughout each LD cycle. As a result, for increased frequency, there is less photoinhibition during the light phase, which results in an increased productivity of the photosystem. With further increasing frequency, the degree of photoinhibition remains constant, while the open-closed dynamics changes from two alternating states to a constant equilibrium value throughout the entire LD cycle. The equilibrium state reached for high frequencies leads to an increased growth rate compared to the very slow alternating state. The maximum growth rate is the one obtained for a constant illumination with the mean light intensity $I_{0} / 2$. This latter point coincides with the theoretical proof of Celikovsk, et al. [6]. The inhibition at $I_{0} / 2$ is of course lower than the inhibition at $I_{0}$. It is less clear that, with a Haldane model, the growth rate at $I_{0} / 2$ is always greater than half the value of the growth rate at $I_{0}{ }^{1}$

$$
\frac{\mu_{H}\left(I_{0}\right)}{2} \leq \mu_{H}\left(\frac{I_{0}}{2}\right)
$$

This effect is even stronger for the portion of the Haldane curve far from the initial tangent i.e. for photoinhibiting light intensities.

This point is important, since it provides an estimation of the flashing effect scale at a given flashing light intensity $I_{0}$. The possible productivity gain between a process where the cells stay too long exposed to a high light and a process where the alternation is fast, is the difference between $B_{s s}\left(I_{0} / 2\right)$ and $B_{s s}\left(I_{0} / 2\right)$. For algae whose photoinhibition is low (at this light intensity), the increase in productivity will then be very low and it will not compensate for the additionally invested mixing energy. This effect can however be stronger when photoinhibition is higher. An efficient management procedure could therefore consist in increasing the agitation velocity only at high light, when the microalgae experience a strong photoinhibition.

It is also interesting to note that the intermediate growth rate for which a plateau, or simply an inflexion point is obtained, corresponds to the Haldane model $B_{s s}\left(I_{0} / 2\right)$, but for which the inhibition term has been doubled $\left(k_{d} / k_{r}\right.$ in the model). This provides a hint on the respective effect of the flashing effect for low and high frequencies.

\section{Conclusion}

The model properties have been derived analytically using a slow/fast approximation. The resulting formulas have a very simple form compared to the exact solution of the Han model [6]. Based on these formulas, a measurement protocol has been proposed to deduce the parameters of the model which relies on the dependency between growth rate and cycle frequency. It can also be derived from the link between growth rate and the maximum light intensity $I_{0}$. Concerning

\footnotetext{
1 This property can be shown by remarking that the Haldane curve $B_{S S}(I)$ is always under its initial tangent for $I=0$. Straightforward geometrical considerations based on Thales Theorem prove this result.
} 
the physiological parameters describing the photosynthetic system, the proposed method for parameter identification varies fundamentally in complexity from other approaches, as proposed by [31] and [23]. Our approach is a simple procedure not implying a tricky numerical integration of the system nor additional off line measurements. This simplifies the experimental protocol and the calculation. In addition, our simple analytical expression allows for a better estimation of the approximation error.

The resulting formulas for the growth rate do not only allow for the determination of the physiological parameters, they also show a characteristic dependency between signal frequency and growth rate which enables an easy experimental validation of the model. The shape of the curve explains the "Flashing Light Effect" and shows that the increase of growth rate with fluctuation frequency is not linear but shows a very characteristic saturation for intermediate frequencies. Only a weak increase in growth rate can be expected with frequency augmentation in this domain. This insight can lead to important consequences for the design of raceways and photobioreactors. The dynamics of nitrogen assimilation and nutrient limited growth play an inferior role under the conditions we were assuming.

The proposed model is suitable to investigate the effect of the hydrodynamic regime on the growth rate of microalgae cultures due to photoinhibition. Regarding biofuel production with raceways and photobioreactors, the light variability on faster time scales is typically caused by mixing. The results from this study give important insights and are a first step in understanding the coupling of physical and biological models. With further research on the Droop-Han Model, the light intensity dynamics at the scale of the microalgae can be optimized by the design of the process and an increased productivity can be achieved using the same total light dose. Increasing efficiency in biomass production by optimized photobioreactors is an important step on the way to the industrial use of microalgae.

Acknowledgements: This work was carried out in the framework of the ARC Nautilus funded by INRIA.

\section{References}

1. J R Benemann. $\mathrm{CO}_{2}$ mitigation with microalgae systems. Energy Conversion and Management, pages 475-479, 1997.

2. O Bernard. Hurdles and challenges for modelling and control of microalgae for CO2 mitigation and biofuel production. Journal of Process Control, 21(10):1378-1389, 2011.

3. O Bernard and J L Gouzé. Transient behavior of biological loop models, with application to the Droop model. Mathematical Biosciences, 127(1):19-43, 1995.

4. O Bernard and J L Gouzé. Nonlinear qualitative signal processing for biological systems: application to the algal growth in bioreactors. Math. Biosciences, 157:357-372, 1999.

5. A P Carvalho, L A Meireles, and F X Malcata. Microalgal reactors: A review of enclosed system designs and performances. Biotechnology Progress, 22(6):1490-1506, 2006.

6. S Celikovsk, D Stys, S Papacek, S Celikovsky, and J Ruiz-Leon. Bilinear system as a modelling framework for analysis of microalgal growth. Kybernetika, 43(1):1-20, 2007.

7. Y Chisti. Biodiesel from microalgae. Biotechnology Advances, 25:294-306, 2007.

8. M R Droop. Vitamin $B_{12}$ and marine ecology. The kinetics of uptake growth and inhibition in Monochrysis lutheri. J. Mar. Biol. Assoc., 48(3):689-733, 1968.

9. M R Droop. 25 Years of Algal Growth Kinetics, A Personal View. Botanica marina, 16:99-112, 1983.

10. P H C Eilers and J C H Peeters. A model for the relationship between light intensity and the rate of photosynthesis in phytoplankton. Ecological modelling, 42(3-4):199-215, September 1988. 
11. P H C Eilers and J C H Peeters. Dynamic behavior of a model for photosynthesis and photoinhibition. Ecological modelling, 69(1-2):113-133, September 1993.

12. B P Han. Photosynthesis-irradiance response at physiological level: A mechanistic model. J Theor Biol, 213:121-127, 2001.

13. B P Han. A mechanistic model of algal photoinhibition induced by photodamage to photosystem-II. Journal of theoretical biology, 214(4):519-27, February 2002.

14. M Huntley and D G Redalje. $\mathrm{CO}_{2}$ Mitigation et Renewable Oil from Photosynthetic Microbes: A New Appraisal. Mitigation and Adaptation Strategies for Global Change, 12:573-608, 2007.

15. K Lange and F J Oyarzun. The Attractiveness of the Droop Equations. Mathematical Biosciences, 111:261-278, 1992.

16. HP Luo and Al-Dahhan. Analyzing and modeling of photobioreactors by combining first principles of physiology and hydrodynamics. Biotechnology \& Bioengineering, 85:382-393, 2004.

17. F Mairet, O Bernard, P Masci, T Lacour, and A Sciandra. Modelling neutral lipid production by the microalga Isochrysis affinis galbana under nitrogen limitation. Biores. Technol., 102:142-149, 2011.

18. F B Metting. Biodiversity and application of microalgae. Journal of Industrial Microbiology and Biotechnology, 17:477-489, 1996.

19. Monod. La technique de culture continue. théorie et applications. Annales de L'intiut Pasteur, 79:390-410, 1950.

20. M Olaizola. Commercial development of microalgal biotechnology: from the test tube to the marketplace. Biomolecular Engineering, 20:459-466, 2003.

21. I Perner-Nochta and C Posten. Simulations of light intensity variation in photobioreactors. Journal of Biotechnology, 131:276-285, 2007.

22. O Pulz. Photobioreactors: production systems for phototrophic microorganisms. Applied Microbiology et Biotechnology, 57:287-293, 2001.

23. B Rehak, S Celikovsky, and S Papcek. Model for Photosynthesis and Photoinhibition: Parameter Identification Based on the Harmonic Irradiation $\mathrm{O}_{2}$ Response Measurement. IEEE Transactions on Automatic Control, 53(Special Issue):101-108, January 2008.

24. T Sari. Averaging for ordinary differential equation and functional differential equations. In I van den Berg and V Neves, editors, The Strength of Nonstandard Analysis, pages 286-305. Springer, 2007.

25. A Sciandra and P Ramani. The limitations of continuous cultures with low rates of medium renewal per cell. J. Exp. Mar. Biol. Ecol., 178:1-15, 1994.

26. B Sialve, N Bernet, and O Bernard. Anaerobic digestion of microalgae as a necessary step to make microalgal biodiesel sustainable. Biotechnol. Advances, 27:409-416, 2009.

27. Prezlin V. Light reaction in photosynthesis. Can. J. Fish Aquat. Sci., 210:1-43, 1981.

28. I Vatcheva, H de Jong, O Bernard, and N J L Mars. Experiment selection for the discrimination of semi-quantitative models of dynamical systems. Artif. Intel., 170:472-506, 2006.

29. C Vejrazka, M Janssen, M Streefland, and R Wijffels. Photosynthetic efficiency of Chlamydomonas reinhardtii in flashing light. Biotechnology and bioengineering, 108(12):29052913, July 2011.

30. R H Wijffels and M J Barbosa. An outlook on microalgal biofuels. Science, 329(5993):796799, Aug 2010.

31. X Wu. A model integrating fluid dynamics in photosynthesis and photoinhibition processes. Chemical Engineering Science, 56(11):3527-3538, June 2001.

\section{Appendix A: Analytical Derivation}

\section{A1. Low Frequency Signal}

Eq. (12) is taken as an expression for the productivity of the photosynthetic system and the mean value during one period is taken. The domain of the integral can be restricted to the light phase of the cycle only, since during the dark phase $B=0$.

$$
\bar{B}_{\text {slow }}=\frac{1}{2 T} \int_{T} B d t
$$


By replacing $A$ by $1-B-C$, and assuming that $\mathrm{A}$ rests in its equilibrium while $C$ is periodically oscillating:

$$
B=\tau \sigma I A
$$

we get:

$$
\bar{B}_{\text {slow }}=\frac{1}{2} \frac{\tau \sigma I_{0}}{1+\tau \sigma I_{0}}\left(1-\frac{1}{T} \int_{T} C d t\right)
$$

Analogously, the differential equation for $C$ can be reformulated

$$
\dot{C}=-\left(k_{d} \tau \frac{\left(\sigma I_{0}\right)^{2}}{1+\tau \sigma I_{0}}+k_{r}\right) C+\frac{\left(\sigma I_{0}\right)^{2}}{1+\tau \sigma I_{0}}
$$

This equation can be easily solved. $C_{0}$ is determined by applying the periodic boarder condition. Several simplification steps lead to the following expression for the integral:

$$
\int_{T} C d t=\frac{C_{0}-\delta(I)}{\epsilon}(1-\gamma(I)+\delta(I) T)
$$

Using expression (40), we get expression (19).

\section{A2. High Frequency Signal}

Under the precondition that the periodicity of the signal is short, we already showed that the dynamics of $C$ is negligible. In contrast, the transition process of $A$ and $B$ has to be taken into account. The expression for the mean growth rate has to account for the entire period, since $B \geq 0$ during the dark phase. The expression for the growth rate turns to:

$$
\bar{B}_{\text {fast }}=\frac{1}{2 T}\left(\int_{0}^{T} B d t+\int_{T}^{2 T} B d t\right)
$$

The first integral being during the light period and the second during the dark period. To solve the integrals, an expression for $A(t)$ has to be determined from the differential equation. By assumption that $C$ is a constant value and with periodical boarder conditions, Eq. (14) can be solved with the result:

$$
\begin{aligned}
& A(t)=\left(A_{0}+\frac{1-C}{1+I_{0} \sigma \tau}\right) e^{\left(-\frac{t}{\tau}-I_{0} \sigma \xi t\right)}-\frac{1-C}{1+I_{0} \sigma \tau}, \text { for } t \in[0, T] \\
& A(t)=\left(A_{T}+1-C e^{\left(-\frac{t-T}{\tau}\right)}\right)-1-C, \text { for } t \in[T, 2 T]
\end{aligned}
$$

With Eq. (44) and (14), periodical boarder conditions and the assumption $\dot{C}=0$, a value for $C$ can be determined to:

$$
C=\frac{k_{d}(\sigma I)^{2}}{2 T k_{r}} \frac{T \eta-T \theta}{\left.\eta^{2}+\frac{k_{d}(\sigma I)^{2}}{2 T k_{r}}(T \eta-T] \theta\right)}
$$

with the expressions:

$$
\eta=\frac{1}{\tau}+\sigma I_{0}, \theta=\frac{\left(1-\alpha_{0}\right)\left(1-\alpha_{I}\right)}{T\left(1-\alpha_{0} \alpha_{I}\right)}, \alpha_{I}=e^{-\left(\frac{1}{\tau}+\sigma I_{0}\right) T}, \alpha_{0}=e^{-\frac{T}{\tau}}
$$


Knowing the value of $C$, the integrals in Eq. (43) can be resolved and $B$ can be formulated as:

$$
\bar{B}_{f a s t}=\frac{\sigma I_{0}}{2} \frac{\eta-\theta+\tau \delta \theta}{\eta^{2}+\frac{k_{d}(\sigma I)^{2}}{2 k_{r}}(\eta-\theta)}
$$

\section{A3. Proof of Monotony}

\section{Low Frequency}

It can be shown, that the function:

$$
\Phi(x, a, b)=\frac{\left(1-e^{-a x}\right)\left(\left(1-e^{-b x}\right)\right)}{x\left(1-e^{-(a+b) x}\right)}
$$

is strictly monotonic decreasing in $x$ and it holds $\Phi(x, a, b)>0$ for $a>0, b>$ $0, x>0$

Further, $\bar{B}_{\text {slow }}(I, T)$ can be defined as follows:

$$
\bar{B}_{\text {slow }}(I, T)=\left(1+\frac{\delta(I)}{k_{r}} \Phi\left(T, k_{r}, \chi\right)\right) \cdot \bar{B}_{S S} / 2
$$

with $\chi=k_{r}+k_{d} \frac{(\sigma I)^{2}}{1+\tau \sigma I}$, consequently, $\bar{B}_{\text {slow }}$ is strictly monotonic decreasing in $T$.

\section{High frequency}

$\bar{B}_{\text {fast }}$ can be written as:

$$
\bar{B}_{\text {fast }}=\frac{\sigma I_{0}}{2} \frac{\eta-\Phi(1 / \tau, \eta, T)+\tau \eta \Phi(1 / \tau, \eta, T)}{\eta^{2}+\frac{k_{d}(\sigma I)^{2}}{2 k_{r}}(\eta-\Phi(1 / \tau, \eta, T))}
$$

Derivation with respect to $T$ yields:

$$
\frac{\partial \bar{B}_{\text {fast }}}{\partial T}=\frac{\eta^{2}\left(\frac{k_{d} \sigma I^{2}}{2 k_{r}}+\sigma I \tau\right)}{\left(\eta^{2}+\frac{\left.k_{d}(\sigma I)^{2}\right)^{2}}{2 k_{r}}(\eta-\Phi(1 / \tau, \kappa, T))^{2}\right.} \cdot \frac{\partial \Phi(1 / \tau, \eta, T)}{\partial T}
$$

Taking into account the parameters, it is clear that the fraction is positive. With the property $\frac{\partial \Phi(1 / \tau, \eta, T)}{\partial T}<0$, it is shown that $\bar{B}_{\text {fast }}$ is strictly monotonic decreasing with respect to $T$. 